\title{
Sudden Death of Cattle Caused by Bibersteinia trehalosi Infection in Korea
}

\author{
Mi-Na Han \\ Hyeon-Seop Byeon ${ }^{1}$ \\ Mun-Hui Chae ${ }^{1}$ \\ Rae-Hoon Jang ${ }^{1}$ \\ Ki-Jeong $\mathrm{Na}^{2, *}$ \\ ${ }^{1}$ Institute of Chungbuk Provincial Veter- \\ inary Service and Research, Cheongju \\ 28153, Korea \\ ${ }^{2}$ Laboratory of Veterinary Laboratory \\ Medicine, College of Veterinary Medicine, \\ Chungbuk National University, Cheongju \\ 28644, Korea
}

Abstract Two cases of sudden death without clinical signs in a four-month-old female Hanwoo (Bos taurus coreanae) and sixteen-month-old female Holstein (Bos taurus taurus) were investigated. Severe pneumonia and septicemia were found on postmortem examination. Petechial and ecchymotic hemorrhages were present in the subcutaneous tissues of the neck region. The lungs remained expanded without collapsing, and the mediastinal lymph nodes were enlarged. A significant number of hemorrhagic bubbles were present in the tracheal lumen. On histopathology, the alveolar blood vessels were moderately congested, and inflammatory cells and multinucleated syncytial cells were found in the alveolar space. The pathogen isolated from the lesion was identified as Bibersteinia trehalosi using MALDI-TOF and 16S rRNA gene and sodA gene sequence analyses. To the best of our knowledge, this is the first report of $B$. trehalosi infection in cattle in Korea.

Key words sudden death, cattle, pneumonia, septicemia, Bibersteinia trehalosi.
*Correspondence: sigol@cbnu.ac.kr

\section{ORCID}

Mi-Na Han:

https://orcid.org/0000-0002-0865-4530

Hyeon-Seop Byeon:

https://orcid.org/0000-0003-1919-483X Mun-Hui Chae:

https://orcid.org/0000-0002-4541-7315

Rae-Hoon Jang:

https://orcid.org/0000-0001-6766-2592

Ki-Jeong Na:

https://orcid.org/0000-0001-6076-3147

Copyright $\odot$ The Korean Society of Veterinary Clinics
Received August 3, 2021 / Accepted October 19, 2021 


\section{Introduction}

Bibersteinia (Pasteurella) trehalosi is a small, non-motile, Gram-negative rod and coccobacilli (4). It belongs to the family Pasteurellaceae and has a genetic relationship with Mannheimia haemolytica. However, B. trehalosi is distinguished from other Pasteurellaceae strains by fermenting trehalose and is distinguished from $M$. haemolytica based on its ability to ferment arabinose or trehalose (6). P. trehalosi was reclassified as $B$. trehalosi using phenotyping identification such as biochemical characterization, 16s rRNA gene sequence analysis, and AFLP typing (4).

B. trehalosi is an important pathogen that causes septicemia and respiratory disease in calves and lambs. Clinical signs include sudden death (mortality rate: 2.5-3.2\%), dullness, fever, frothy discharge from the mouth, exhaustion, and poor physical condition (2). However, these symptoms are difficult for livestock managers to detect because animal death occurs within 6 to 8 hours after the onset of clinical signs (5). Gross pathology in affected cattle includes severe pneumonia and septicemia (2). The epicardium, cardiac muscle, and lungs show widespread hemorrhage, the lymph nodes are enlarged and show hemorrhage, and the lungs show severe congestion and edema $(3,5)$. Clinical symptoms are more severe if antibiotics are ineffective or the host is infected with other respiratory pathogens such as Pasteurella multocida and Mycoplasma bovis (9).
Gene sequence analysis can improve our understanding of bacterial phylogenetic relationships and identify numerous species (13). By sequencing the $16 \mathrm{~S}$ rRNA gene and the sodA gene, $B$. trehalosi can be quickly and accurately distinguished from other species belonging to the family Pasteurellaceae $(8,13)$. A phylogenetic analysis using $16 \mathrm{~S}$ rRNA gene is required because traditional metabolism-based methods are unable to accurately distinguish $B$. trehalosi from other members of the family Pasteurellaceae (12). Furthermore, the sodA sequence shows higher variability than the 16S rRNA gene, and its divergence is especially helpful to differentiate closely related species or subspecies of Pasteurella that cannot be differentiated with equal confidence via the 16S rRNA gene $(8,13)$.

Peracute to acute fatal pneumonia and septicemia induced by $B$. trehalosi are important diseases causing sudden death in cattle. B. trehalosi-related cases have been reported in Europe and the United States $(5,7,14)$. In Korea, however, disease associated with $B$. trehalosi infection has never been reported. Therefore, in this study, we report two cases of cattle with $B$. trehalosi-associated severe pneumonia and septicemia occurring in Korea.

\section{Case}

Two cases of sudden death without clinical signs in cattle are reported. Each carcass was presented for postmortem
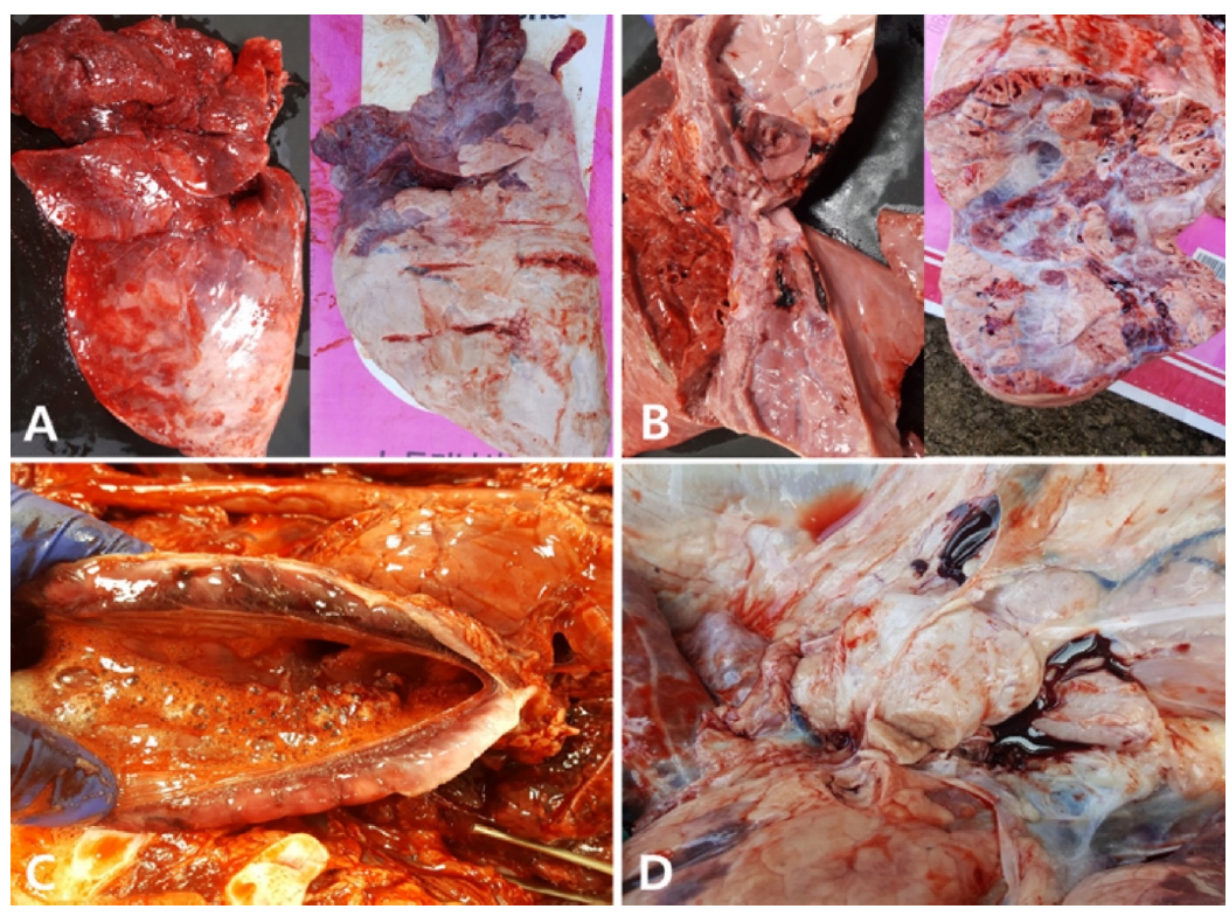

Fig. 1. Postmortem examination. The anterior parts of the lung lobes were discolored and consolidated (A). The lobular connective tissue of the caudal lobes had accumulated air that created spaces (B). Hemorrhagic bubbles were present in the tracheal lumen (C). The mediastinal lymph nodes were edematous and pale (D). The left side $(A, C)$ is the case of a Hanwoo heifer, and the right side $(B, D)$ is the case of a Holstein heifer. 
examination at the Institute of Chungbuk Provincial Veterinary Service and Research. A four-month-old Hanwoo and a sixteen-month-old Holstein heifer were presented in January 2018 and August 2017, respectively. The farmers did not recognize any signs of illness on the day before death.

\section{Pathological examination}

On postmortem examination, petechial and ecchymotic hemorrhage were present in the subcutaneous tissues surrounding the trachea, esophagus, and cervical blood vessels of the neck. The cranial and middle lung lobes were atelectatic and had a rubbery-like hardness and dark red color. In contrast, the caudal lung lobes were excessively enlarged (Fig. 1A). The cut surface of the caudal lobes was dark red color in (Fig. 1B). The tracheal lumen was filed with hemorrhagic bubbles (Fig. 1C). The mediastinal lymph nodes were 2-3 times larger than their normal size, and they were edematous and pale on cut section (Fig. 1D). On histopathological examination of the lungs, the alveolar blood vessels of both cranial lobes were diffusely moderately congested. The alveolar space contained a large amount of eosinophilic material, fibrin, neutrophils, and alveolar macrophages and occasional multinucleated syncytial cells (Fig. 2A, B).

\section{Laboratory examination}

For bacteriological examination, the lung samples were cultured. No hemolysis was present, but round, white colonies were identified growing on blood agar. These bacterial colonies were identified as $B$. trehalosi using MALDI-TOF (microflex ${ }^{\circledR}$, BRUKER, USA) and 165 ribosomal RNA (16S rRNA) gene and superoxide dismutase ( $\operatorname{sod} A)$ gene sequencing. The $16 \mathrm{~S}$ rRNA primers $F$ ( $5^{\prime}$-AGAGTTTGATCCTGGCTCAG-3') and R ( $5^{\prime}$-CTTGTGCGGGCCCCCGTCAATTC-3') were used for the amplification of the $16 \mathrm{~S}$ rDNA gene fragment. And,
sodA primers d1 (5'-CCITAYICITAYGAYGCIYTIGARCC) and d2 (5'-ARRTARTAIGCRTGYTCCCAIACRTC-3') were used to amplify the sodA gene fragment (8). The sequences of the isolates were deposited in GenBank (MK920113 and MK920112 in 16s rRNA gene; MZ558782 and MZ558783 in sodA gene). Multiple alignment of the partial 16s rRNA gene (1325 bp) and sodA gene (465 bp) sequences were performed with MEGA X software. A phylogenetic tree based on these genes showed that the two isolates were $B$. trehalosi (Fig. 3).

An antibiotic susceptibility test with disk diffusion method was also performed. The types of disks were amikacin, cefalexin, enrofloxacin, gentamicin, kanamycin, neomycin, penicillin, and streptomycin. Both isolates were susceptible to enrofloxacin and cefalexin and were resistant to other antibiotics.

\section{Discussion}

This study reports the first cases of $B$. trehalosi infection in South Korea. A typical feature of $B$. trehalosi-related infectious disease is sudden death of an animal without any clinical signs. However, severe pneumonia can be identified on postmortem examinations. Until 2012, there were relatively few cases of $B$. trehalosi infections among cattle in Europe (2). Since 2012, additional case reports of peracute to fatal pneumonia in cattle caused by $B$. trehalosi infections in North America have been published (5). Although studies on cases of $B$. trehalosi infection in Europe and North America have been published for a long time, no cases have been reported in Korea. These are the first two cases of disease associated with $B$. trehalosi infection in cattle in Korea.

Cattle that were acting normally one day were suddenly found dead the next day. The postmortem findings showed severe pneumonia and septicemia including hemorrhage
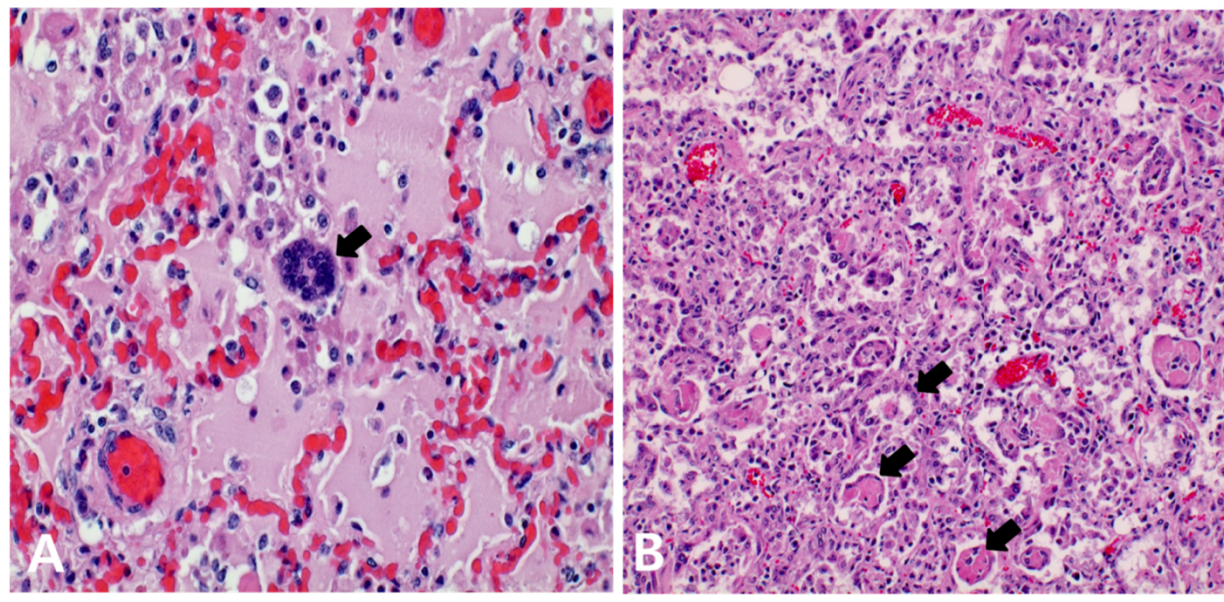

Fig. 2. Histopathological examination. The alveolar blood vessels were moderately congested, and the alveoli were filled with a large amount of eosinophilic material and fibrin and moderate numbers of neutrophils and alveolar macrophages. Syncytial cell (arrow) with a mass of cytoplasm containing several basophilic nuclei, $\times 400$ (A). Necrotized neutrophils, foamy macrophages, and syncytial cells (arrow) present in the bronchial lumen, $\times 200$ (B). Hematoxylin and eosin stain. 
16s rRNA gene

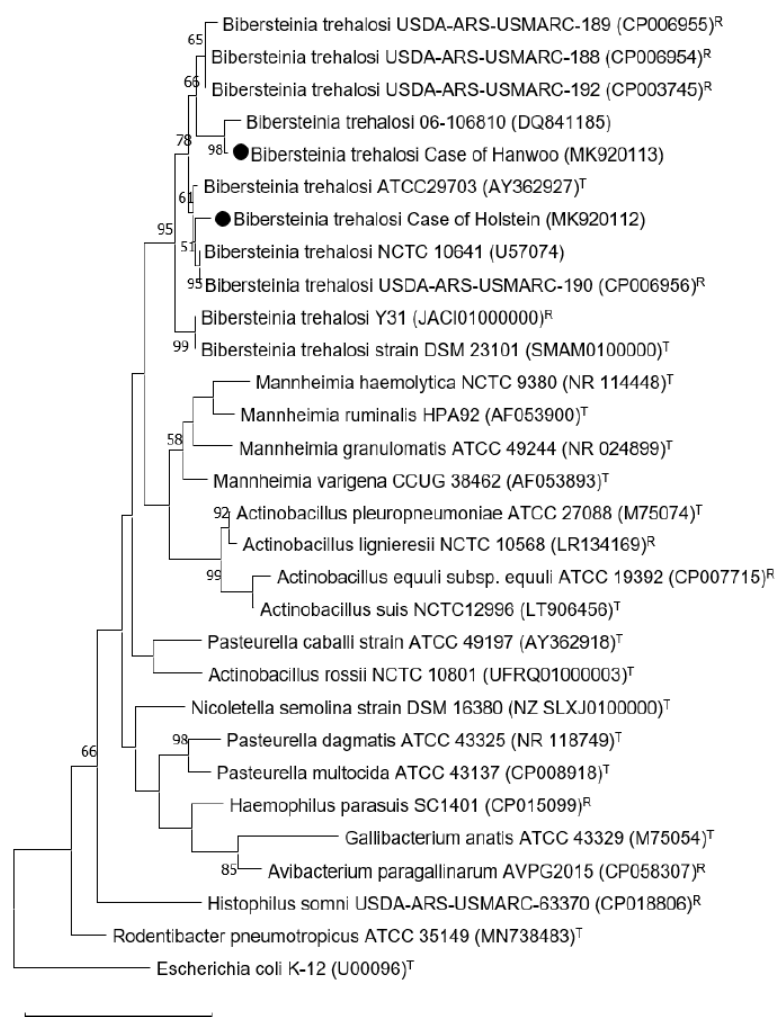

0.1

\section{SodA gene}

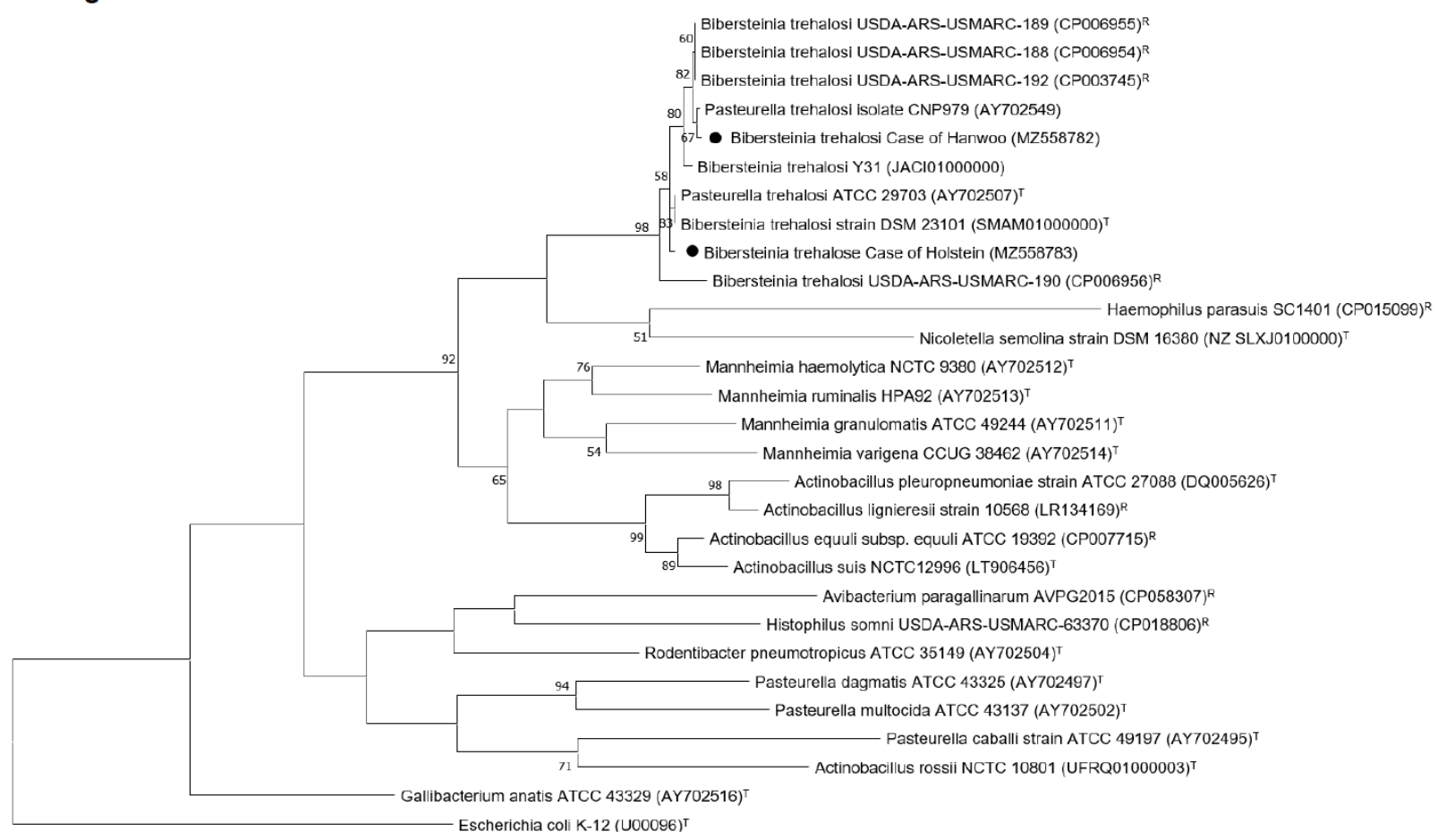

Fig. 3. Phylogenetic tree of the family Pasteurellaceae based on $16 \mathrm{~S}$ rRNA and sodA gene sequences by maximum-likelihood analysis with a bootstrap method (1000 replicates). Reference strains were downloaded from National Center for Biotechnology Information (NCBI). Isolates sequenced in the present study are indicated by black circles. Only bootstrap values of greater than $50 \%$ are indicated. The scale length corresponds to 0.1 nucleotide substitutions per site. 
on the heart and fascia (16). The Animal and Plant Health Agency in the United Kingdom published disease surveillance reports of cattle that were systemically infected with $B$. trehalosi in 2015. This may explain the postmortem lesions found in the infected cattle with revealed widespread hemorrhages within the cardiac muscle and severe pneumonia. In addition, the liver was enlarged, the lymph nodes and spleen were hemorrhagic, and yellow fluid was present in the abdomen (3). Multinucleated syncytial cells were observed on histopathological examination. These histopathological changes in the lungs indicate concurrent or previous exposure to bovine respiratory syncytial virus (BRSV) (1). B. trehalosi infections are more severe after normal lung defenses have been compromised by another respiratory pathogen. In sheep, respiratory infection pathogens (i.e., adenoviruses, RSV, and Mycoplasma ovipneumoniae.) do not cause serious disease when a single infection is present. However, when combined with $B$. trehalosi infection, the morbidity and mortality are increased (11).

In these two cases, the isolates were susceptible to enrofloxacin and cephalexin on antibiotic susceptibility tests. On cattle farms with $B$. trehalosi infection, prompt antibiotic treatment of calves that are suspected having the infection is necessary to prevent additional losses (3). Therefore, selection of and treatment with appropriate antibiotics using antibiotic susceptibility testing can prevent the spread of this disease. The 16S rRNA gene consensus sequence of the case in the Hanwoo heifer had $99.01 \%$ similarity with the GenBank accession number DQ841185 and $97.36 \%$ similarity with the accession number AY362927. And that of the case in the Holstein heifer had $98.83 \%$ similarity with the accession number AY362927 and $97.82 \%$ similarity with the accession number U57074. Furthermore, the sodA gene consensus sequence of the case in the Hanwoo heifer had $99.65 \%$ similarity with the GenBank accession number AY702549 and 99.38\% similarity with the accession number CP003745, CP006954 and CP006955. And that in the case in the Holstein heifer had 99.54\% similarity with the accession number AY702507 and 98.63\% similarity with the accession number JACI01000000. Phylogenetic analysis revealed that the isolates in these cases were identical to the $B$. trehalosi as supported by bootstrap values of $95 \%$ in the $16 \mathrm{~S}$ rRNA gene and $98 \%$ in the sodA gene (Fig. 3). The analysis of sodA gene sequence provides a fast and accurate method for species identification, and the high discriminant power associated with $\operatorname{sod} A$ gene variability has helped to recognize other members of the Pasteurellaceae, which cannot distinguish closely related species or subspecies using the 16S rRNA gene sequencing (Fig. 3).

B. trehalosi is one of the most commonly isolated patho- gens in ovine pneumonia and is associated with high morbidity and mortality rates in domestic sheep $(4,10,15)$. However, recently, there has been an increase in reports of sudden death in cattle and goats due to this disease in the United States and the United Kingdom $(5,7,14)$.

\section{Conclusions}

This is the first report of $B$. trehalosi infection in cattle in South Korea. The characteristics of this disease include sudden death without clinical signs in cattle. On gross necropsy, fatal pneumonia and septicemia were observed. Congested alveolar blood vessels, inflammatory cells, and multinucleated syncytial cell in the alveolar space were observed on histopathological examination. Bacterial colonies were isolated from the lungs and identified as $B$. trehalosi using MALDI-TOF and sequence analysis of the 16S rRNA gene and sodA gene.

\section{Acknowledgements}

This study was conducted with the budget, a head of experiment and research (20703), of the Institute of Chungbuk Provincial Veterinary Service and Research.

\section{Conflict of Interest}

The authors declare that they have no conflict of interest.

\section{References}

1. Al-Sadrani AA, Abdelsalam EB. Histological evidence of a respiratory syncytial virus infection in pneumonic lungs of sheep in Al-Quasim area, Kingdom of Saudi Arabia. Bulg J Vet Med 2010; 13: $186-189$.

2. Alegre M, Nanni M, Fondevila N. Development of a multiplex polymerase chain reaction for the differentiation of bovine herpesvirus-1 and -5. J Vet Med B Infect Dis Vet Public Health 2001; 48: 613-621.

3. Bibersteinia trehalosi infection in cattle and sheep. Vet Rec 2015; 176: 195-198.

4. Blackall PJ, Bojesen AM, Christensen H, Bisgaard M. Reclassification of [Pasteurella] trehalosi as Bibersteinia trehalosi gen. nov., comb. nov. Int J Syst Evol Microbiol 2007; 57(Pt 4): 666-674.

5. Cortese VS, Braun DA, Crouch D, Townsend C, Zukowski B. Case report - peracute to acute fatal pneumonia in cattle caused by Bibersteinia trehalosi. Bov Pract 2012; 46: 138-142.

6. Dassanayake RP, Call DR, Sawant AA, Casavant NC, Weiser GC, Knowles DP, et al. Bibersteinia trehalosi inhibits the growth of 
Mannheimia haemolytica by a proximity-dependent mechanism. Appl Environ Microbiol 2010; 76: 1008-1013

7. Davies RL, Whittam TS, Selander RK. Sequence diversity and molecular evolution of the leukotoxin (IktA) gene in bovine and ovine strains of Mannheimia (Pasteurella) haemolytica. J Bacteriol 2001; 183: 1394-1404.

8. Gautier AL, Dubois D, Escande F, Avril JL, Trieu-Cuot P, Gaillot O. Rapid and accurate identification of human isolates of Pasteurella and related species by sequencing the sodA gene. J Clin Microbiol 2005; 43: 2307-2314.

9. Gilmour NJ. Pasteurellosis in sheep. Vet Rec 1978; 102: 100-102.

10. Kehrenberg C, Meunier D, Targant H, Cloeckaert A, Schwarz S, Madec JY. Plasmid-mediated florfenicol resistance in Pasteurella trehalosi. J Antimicrob Chemother 2006; 58: 13-17.

11. Miller MW, Knowles DP, Bulgin MS. CAST Commentary QTA 2008-1. Pasteurellosis transmission risks between domestic and wild sheep. Ames: Council for Agricultural Science and Technology; 2008. Report No.: 2007-31100-06019/ISU Project No. 413-
40-02 and Grant No. 2008-38902-19327.

12. Miller MW, Hause BM, Killion HJ, Fox KA, Edwards WH, Wolfe LL. Phylogenetic and epidemiologic relationships among Pasteurellaceae from Colorado bighorn sheep herds. J Wildl Dis 2013; 49: 653-660.

13. Public Health England. UK standards for microbiology investigations. Identification of Pasteurella species and morphologically similar organisms. Harlow: Public Health England. 2015. 28 p.

14. Spagnoli S, Reilly TJ, Calcutt MJ, Fales WH, Kim DY. Subcutaneous botryomycosis due to Bibersteinia trehalosi in a Texas Longhorn steer. Vet Pathol 2012; 49: 775-778.

15. Szeredi L, Rausch F, Szeleczky Z, Jánosi S. High mortality caused by Bibersteinia trehalosi septicaemia in adult sheep - a case report. Acta Vet Hung 2018; 66: 509-517.

16. Watson PJ, Scholes SF. Bibersteinia trehalosi necrotising hepatitis associated with sudden death in an adult cow. Vet Rec 2010; 167: 100-102. 